doi: http://dx.doi.org/10.5892/RUVRV.91.89106

\title{
ANÁLISE DA INFLUÊNCIA DE DETERMINADOS FATORES SOBRE O ESTADO NUTRICIONAL DE CRIANÇAS RESIDENTES EM COMUNIDADES RURAIS DE DIAMANTINA-MG
}

\author{
Paola A. A. FERREIRA ${ }^{1}$ \\ Gustavo H.B. PASCOAL ${ }^{2}$ \\ Milton C. RIBEIRO ${ }^{3}$ \\ Emerson C. BODEVAN ${ }^{4}$ \\ Daisy R.F. FERNANDES² \\ Sheyla R.R.MARTINS 3 \\ Ana Paula F.C. VANZELA ${ }^{3}$ \\ Marcos L.P. PINHEIRO5
}

Leida Calegário de OLIVEIRA ${ }^{3}$

RESUMO: O aleitamento materno é considerado um dos pilares fundamentais para a promoção e proteção da saúde em todo o mundo. Apesar do amplo reconhecimento de sua importância, na maioria dos países as taxas de amamentação exclusiva ainda são baixas e a duração, insatisfatória. O presente estudo buscou caracterizar o estado nutricional de crianças na faixa etária entre cinco e nove anos e comparar com as variáveis tempo de amamentação exclusiva, renda familiar, escolaridade da mãe e número de membros na família destas crianças, residentes em nove comunidades rurais de Diamantina - MG, Brasil. Para avaliação do estado nutricional, foram utilizadas medidas antropométricas de peso e estatura, construídos índices e estabelecidos pontos de corte segundo as curvas do National Center for Health Statistic - NCHS. Para obtenção das variáveis foi utilizado um questionário desenvolvido pela equipe. Cento e quarenta e nove crianças foram

${ }^{1}$ Departamento de Nutrição da Universidade Federal dos Vales do Jequitinhonha e Mucuri - UFVJM, Diamantina-MG; ${ }^{2}$ Departamento de Enfermagem da UFVJM, Diamantina-MG; ${ }^{3}$ Departamento de Farmácia-Bioquímica da UFVJM, Diamantina-MG; ${ }^{4}$ Departamento de Matemática; ${ }^{5}$ Departamento de Ciências Básicas da UFVJM, Diamantina-MG.

Endereço para correspondência: $\operatorname{Dr}^{\mathrm{a}}$ Leida Calegário de Oliveira, Professora Adjunto, Departamento de Farmácia, Faculdade de Ciências Biológicas e da Saúde, Universidade Federal dos Vales do Jequitinhonha e Mucuri, Campus Juscelino Kubitschek - Rodovia MGT 367 - Km 583, nº 5000, Alto do Jacuba. CEP 39.100-000 - Diamantina - MG - tel: (38) 3532.6000 Email: leida@ufvjm.edu.br. 
sorteadas aleatoriamente (n calculado para atender aos critérios: significância 0,05 e poder do teste 0,9). Os resultados mostraram que 76,87\% das crianças encontrava-se eutrófica, $19,73 \%$ encontrava-se em risco nutricional (baixo peso e desnutridas) e 3,4\% encontrava-se com sobrepeso. Os dados revelaram que em crianças que não foram amamentadas exclusivamente até os seis meses de idade, houve predomínio de risco nutricional. Observou-se ainda que o estado nutricional é afetado pelo número de membros na família e escolaridade da mãe. O risco nutricional está atingindo populações rurais, formadas principalmente por famílias de baixa renda e com dificuldades de acesso a serviços de saúde, tornando necessárias ações para reverter tal quadro.

Palavras-chave: estado nutricional, amamentação exclusiva, renda familiar, Vale do Jequitinhonha.

ABSTRACT: Breast milking is worldwide considered one of the fundamental pillars for health promotion and protection. In spite of its generally recognized importance, rates of breast milking alone are still low in most countries, and its lasting is unsatisfactory. This study aimed to evaluate the nutritional condition of 5-9 years old children in correlation with the variables duration of breast milking alone, family income, mother's literacy and number of family members. The study was performed with children dwelling in nine rural communities from Diamantina-MG, Brazil. In order to evaluate the nutritional condition, it was utilized anthropometric measures of weight and height. Indexers were constructed and cut-off values were established in accordance to the curves of the National Center for Health Statistic - NCHS. The variables were obtained by means of a questionnaire developed by the authors. A number of 149 children were randomly choosen (n value for a significance of 0.05 and test power of 0.9). Results showed that $76.87 \%$ of the children presented normal nutritional conditions, while $19.73 \%$ were in nutritional risk since they were underweight and presented evidences of malnutrition, whereas $3.4 \%$ were overweight. Data indicated that the nutrition risk were predominant for children who were not exclusively breastmilked until the $6^{\text {th }}$ month. It was also observed that the nutritional condition is influenced by the number of family members and by mother's literacy. Therefore, rural populations, mainly composed by low-income families with impaired healthy assistance, are being exposed to nutritional risk, whereas some reverting intervention is required

Key-words: nutritional conditions, breast milking alone, family income, Jequitinhonha Valley.

\section{INTRODUÇÃO}

Os Vales do Jequitinhonha e do Mucuri fazem parte de uma macrorregião que corresponde a aproximadamente $33 \%$

\begin{abstract}
do estado de Minas Gerais, sendo considerada como uma das regiões economicamente mais carentes do Brasil. Possui uma população predominantemente rural que se ocupa basicamente da
\end{abstract}


agricultura de subsistência, extrativismo e mineração. Em países em vias de desenvolvimento, onde a prevalência de casos de desnutrição é alta, especialmente em crianças, torna-se necessária uma constante vigilância nutricional de forma a identificar possíveis distúrbios nutricionais como o déficit no crescimento ou até mesmo a obesidade, o que pode estar relacionado com alguma doença e/ou com as condições sociais desfavoráveis (BISCEGLI et al., 2007).

A desnutrição na infância em termos populacionais é uma doença de origem multifatorial e complexa, que tem suas raízes na situação de pobreza. Ocorre quando o organismo não recebe os nutrientes necessários para o seu metabolismo fisiológico, devido à falta de aporte ou problema na utilização do que lhe é ofertado. Assim sendo, na maioria dos casos, a desnutrição é o resultado de uma ingestão insuficiente de alimentos, ou fome, associada ou não a outras doenças (CAUÁs, et al., 2006).

A avaliação do estado nutricional de crianças é considerada como um instrumento importante na aferição das condições de saúde e de vida de uma população. A análise de estudos sobre a situação nutricional da população brasileira indica que crianças pertencentes ao estrato rural, apresentam situação nutricional desfavorável em relação àquelas de estrato urbano, em decorrência de piores condições de vida. Apesar da queda importante na prevalência da desnutrição infantil brasileira observada entre os três estudos realizados por Monteiro et al.(1995, 1997a, 1997b), esta redução se deu de forma diferenciada no território nacional, intensificando-se as desigualdades entre as regiões brasileiras (Sul e Sudeste versus Norte e Nordeste), entre níveis sócio-econômicos (pobres e ricos) e entre estratos urbano e rural (GUIMARÃES et al. 1999). Segundo Monteiro (1995), a desnutrição medida pelo retardo do crescimento infantil, também assume grandes proporções no país e está presente em todas as regiões e áreas. Neste contexto, as populações rurais são sempre mais atingidas, do que as urbanas.

Muitas investigações têm buscado aprofundar o conhecimento sobre a interferência de fatores econômicos, sociais e biológicos na estatura das crianças, pelas situações observadas em diferentes 
contextos e pela grande magnitude com que apresentaram (OLIVEIRA, et al., 2007).

Segundo Post et al.(1998) crianças de mais baixa renda apresentam prevalências de déficits de peso/idade e comprimento/idade de cerca de 10\%, enquanto que nas categorias de renda superior, estes déficits são de 3\% ou menos. Por outro lado, o sobrepeso apresentou uma tendência crescente com o acréscimo do nível de renda familiar.

A maioria dos estudos sobre avaliação nutricional realizados no século passado se concentrou nos aspectos da desnutrição. Atualmente, nos países desenvolvidos e naqueles em vias de desenvolvimento que se encontram no estágio de transição nutricional (entre os quais o Brasil), verificase redução na prevalência da desnutrição e predomínio do excesso de peso. Assim, são verificados dois extremos da má nutrição desnutrição pela carência e obesidade pelo excesso - que compartilham do mesmo cenário (MARQUES, et al., 2006).

A desnutrição na infância, expressa pelo comprometimento do crescimento linear e/ou ponderal, é ainda um dos principais problemas de saúde enfrentados pelos países em vias de desenvolvimento. No Brasil, a tendência de declínio da desnutrição na infância também está registrada. No entanto, as regiões mais pobres ainda que tenham experimentado as mais elevadas taxas de declínio da desnutrição, ainda continuam a exibir as mais altas prevalências de déficits antropométricos $(<-2 \mathrm{DP}$ do padrão NCHS). Assim, apesar da evolução favorável dos indicadores de saúde e nutrição na infância brasileira, principalmente no que diz respeito ao aleitamento materno, a prevalência da desnutrição em algumas áreas geográficas não acompanhou essa tendência. Observa-se grandes disparidades na distribuição entre as regiões, municípios e áreas urbanas e rurais de um mesmo estado

0 aleitamento materno é considerado um dos pilares fundamentais para a promoção e proteção da saúde das crianças em todo o mundo. A superioridade do leite humano como fonte de alimento, de proteção contra doenças e de afeto fazem com que especialistas do mundo inteiro recomendem a amamentação exclusiva por 4-6 meses de vida do bebê e complementado até pelo menos o final do primeiro ano de vida (SILVA 
et al., 2006). Apesar do amplo reconhecimento da importância do leite materno, na maioria dos países as taxas de amamentação exclusiva ainda são baixas, e a sua duração também é insatisfatória (FALEIROS et al., 2005).

O crescimento saudável é alcançado com uma alimentação adequada. $\mathrm{Na}$ fase inicial da vida, o leite humano é indiscutivelmente o alimento que reúne as características nutricionais ideais, com balanceamento adequado de nutrientes, além de desenvolver inúmeras vantagens imunológicas e psicológicas, importantes na diminuição da morbidade e mortalidade infantil.

O efeito da amamentação sobre o crescimento infantil foi estudado por diversos autores, que constataram que as crianças amamentadas ao seio apresentavam um maior crescimento em relação às amamentadas com fórmulas (DEWEY, 2003).

Nos dias atuais, para se estudar os níveis de saúde de uma determinada população, no sentido de enfatizar a prevenção primária e promoção da saúde, deve-se observar as características biológicas e sociais que estão presentes na mesma. Ressalta-se que os estudos populacionais de crescimento são elaborados a partir dos referenciais de altura/idade e peso/idade. Dentre estes referenciais, o mais utilizado é o do National Center for Health Statistics - NCHS, até por ter sido adotado pela Organização Mundial da Saúde - OMS como curva adequada/referencial internacional para crescimento estatural e observação dos níveis de saúde (FROTA et al., 2007).

Tendo em vista os aspectos apresentados acima, esse estudo teve como objetivo principal traçar o perfil do estado nutricional de crianças das comunidades rurais de Diamantina-MG, comparando a influência da amamentação exclusiva, renda familiar, escolaridade da mãe, número de membros na família e tempo para interrupção da amamentação em função do estado nutricional.

\section{MATERIAL E MÉTODOS}

Este trabalho de extensão em interface com a pesquisa foi desenvolvido por docentes e acadêmicos da Universidade Federal dos Vales do Jequitinhonha e Mucuri 
(UFVJM), entre famílias residentes nas comunidades rurais do município de Diamantina-MG e que apresentavam em sua constituição, crianças entre cinco e nove anos de idade.

Inicialmente, foi feito um levantamento em todas as escolas (públicas e privadas) das comunidades rurais, dos nomes e endereços das crianças na faixa etária estabelecida. A Secretaria Municipal de Educação de Diamantina/MG, a Secretaria de Estado da Educação de Minas Gerais, através da Superintendência Regional de Ensino de Diamantina-MG bem como os diretores das escolas privadas, foram consultados e consentiram na realização desta busca de dados nas escolas. Posteriormente, as escolas receberam uma carta, informando sobre os objetivos do trabalho e solicitando o fornecimento destes dados iniciais para um sorteio, que selecionou as crianças a serem avaliadas. $\mathrm{O}$ número de participantes foi instituído, de forma a atender aos critérios de significância de 0,05 e poder do teste de 0,9 .

Foi realizado um encontro com as mães ou responsáveis legais das crianças nas comunidades rurais, onde os mesmos foram orientados sobre o propósito do trabalho, o tipo e o tempo de duração dos procedimentos e a garantia de sigilo, entre outras orientações necessárias. Após a decisão em participar, foram assinados os Termos de Consentimento Livre e Esclarecido - TCLE. Nestes encontros, foram agendados os dias e locais onde o projeto seria realizado.

Para o desenvolvimento do trabalho, a equipe elaborou um questionário, contendo questões relativas a aspectos sócioeconômicos e determinantes do estado nutricional das crianças. Para os primeiros, foram analisados a renda familiar, o nível de escolaridade da mãe, o número de membros da família e utilizados os Critério de Classificação Econômica Brasil (CCEB) da Associação Brasileira de Empresas de Pesquisa (ABEP). Para os demais, foi investigada a duração da amamentação exclusiva e o momento em que ocorreu a interrupção da amamentação, em função do estado nutricional atual das crianças.

Para os dados antropométricos, foi utilizada uma balança portátil digital (marca Tanita - precisão de 0,1 Kg) para a obtenção da medida do peso e utilizado um antropômetro (marca Altura Exata, de 205 $\mathrm{cm}$ ), para a obtenção da medida da estatura. 
Para a construção dos índices antropométricos (peso/idade - P/I, peso/altura - P/A e altura/idade - A/I), foi realizada a combinação entre o peso, a estatura, a idade e o sexo das crianças.

Os pontos de corte estabelecidos para a classificação nutricional foram aqueles utilizados pelas curvas do National Center for Health Statistics - NCHS, que classifica como situação de desnutrição valores percentis abaixo de 3 , situação de baixo peso valores percentis $\geq 3$ e $<10$, situação de eutrofia valores percentis entre $\geq 10 \mathrm{e}<97 \mathrm{e}$ situação de sobrepeso, valores percentis $\geq$ 97, que foram designados pelas letras $\mathrm{A}, \mathrm{B}, \mathrm{C}$ e $\mathrm{D}$ respectivamente.

Para testar se havia diferença significativa entre o tempo mediano de amamentação exclusiva com relação aos grupos de estado nutricional [desnutrição (A), baixo peso (B), eutrofia (C), sobrepeso (D), foi utilizado o teste, não-paramétrico, de Wilcoxon para comparações múltiplas e para testar se havia alguma dependência (relação) significativa entre renda familiar, escolaridade da mãe, número de membros na família e interrupção da amamentação em função do estado nutricional, foi utilizado o teste exato de Fisher (não-paramétrico). Para as análises estatísticas, os programas computacionais utilizados foram EPI-INFO versão 6.04 e R.

Esta pesquisa foi realizada após aprovação pelo Comitê de Ética em Pesquisa da Universidade Federal dos Vales do Jequitinhonha e Mucuri, e somente participaram do estudo as famílias que assinaram o Termo de Consentimento Livre e Esclarecido (TCLE) aceitando a participação no estudo.

\section{RESULTADOS E DISCUSSÃO}

O presente estudo avaliou cento e quarenta e nove crianças entre cinco e nove anos de idade, residentes em comunidades rurais de Diamantina-MG: Desembargador Otoni (24), Extração (13), Guinda (7), Inhaí (27), Mendanha (7), Planalto de Minas (12), São João da Chapada (21), Senador Mourão (31) e Sopa (7).

Observou-se que as crianças estudadas eram predominantemente de famílias de baixa renda e a principal fonte de renda é a lavoura, destacando-se também a prática da Agricultura Familiar predominantemente de subsistência.

Com o objetivo de estimar a proporção de crianças em cada uma das 
faixas de estado nutricional, fez-se uma análise dos dados obtidos, considerando um nível de confiança de $95 \%$ e segundo o parâmetro $\mathrm{P} / \mathrm{E}$, observou-se que $76,87 \%$ das crianças era eutróficas (estado nutricional C). Pôde-se observar uma proporção de $14,29 \%$ de crianças com baixo peso, denotando um risco à desnutrição (estado nutricional $\mathrm{B}$ ), devido provavelmente às más condições de moradia, alimentação isenta ou carente de nutrientes, condições socioeconômicas desfavoráveis e ausência de água tratada (foco de parasitoses e outras doenças), presentes nas comunidades rurais estudadas. Nos valores percentis extremos, observou-se uma proporção de 5,44\% de crianças desnutridas e de 3,40\% de crianças em situação de sobrepeso. A classificação do estado nutricional das crianças avaliadas neste trabalho pode ser observada na figura 1.

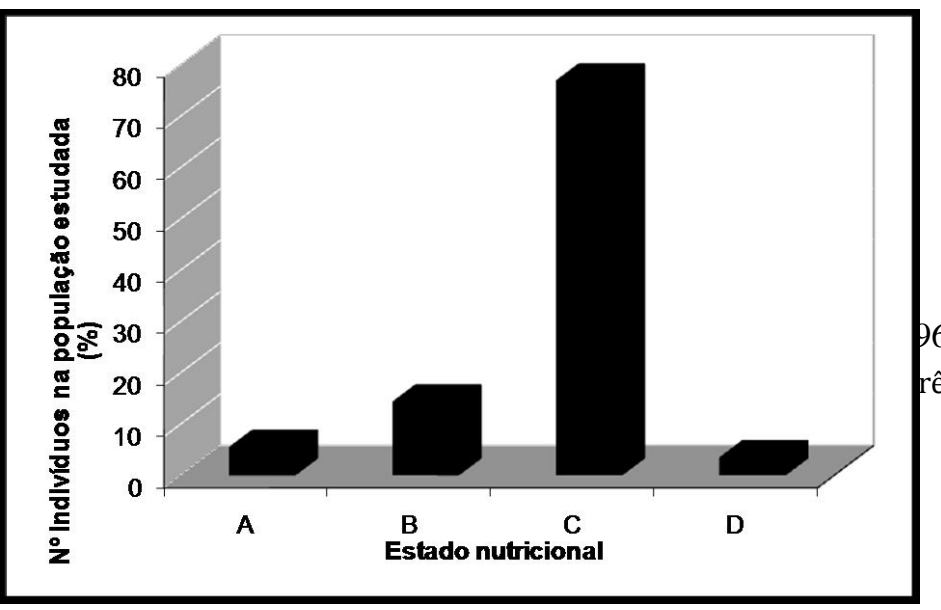

Figura 1 - Relação do estado nutricional e porcentagem de crianças na faixa etária de cinco a nove anos das comunidades rurais de Diamantina/MG, 2007.

Corroborando nossos resultados, Castro et al. (2005) verificaram a classificação do estado nutricional de crianças para os índices $\mathrm{P} / \mathrm{I}, \mathrm{P} / \mathrm{E}$ para os quais foram encontrados valores de 59,8\% e $70,2 \%$ de eutrofia, respectivamente. No entanto, $27,6 \%$ e $19,5 \%$ das crianças encontrava-se em risco para desnutrição, substancialmente maior que o esperado na distribuição normal, na qual 13,6\% de indivíduos estão situados entre -2 e -1 desvio-padrão.

Já no estudo de Burlandy \& Anjos (2007), realizado com crianças de 7 a 10 anos de idade nas regiões rurais e urbanas do Nordeste e Sudeste do Brasil, o resultado encontrado foi diferente, a desnutrição por déficit de peso foi bem baixa e, na verdade, pode-se dizer que não havia desnutrição, visto que as freqüências de valores estavam

ês Corações, v. 9, n. 1, p. 89-106, jan./jul. 2011 
perto do esperado para uma população eutrófica. Por outro lado, a prevalência de sobrepeso foi relativamente alta $(9,5 \%)$, sendo semelhante para meninos e meninas $(9,4 \%$ e $9,7 \%$, respectivamente).

Após estimar o estado nutricional do grupo,-fez-se uma análise comparativa dos tempos de amamentação exclusiva em

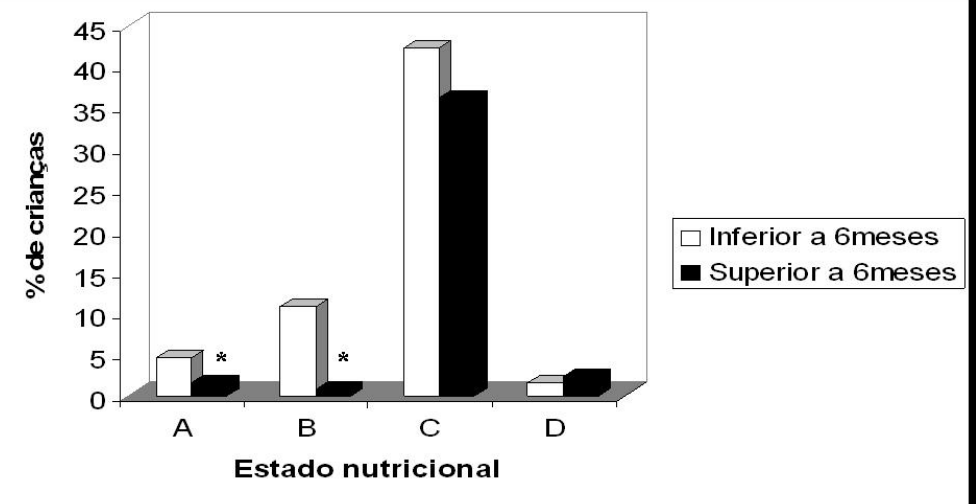
relação aos grupos de estado nutricional e observou-se que houve diferença estatisticamente significativa, apenas entre os grupos A e B. O p-valor encontrado na Figura 2 - Relação entre estado nutricional e porcentagem de crianças na faixa etária de cinco a nove anos residentes nas comunidades rurais de Diamantina/MG, comparado ao tempo de amamentação exclusiva, 2008. comparação desses grupos foi de 0,037 . Na comparação entre os outros grupos (eutrofia e sobrepeso), os p-valores foram superiores a 5\%, indicando que não há diferença significativa entre tempos de amamentação exclusiva e eutrofia e sobrepeso. 0 resultado encontrado pode ser observado nas Figuras 2 e 2.1.

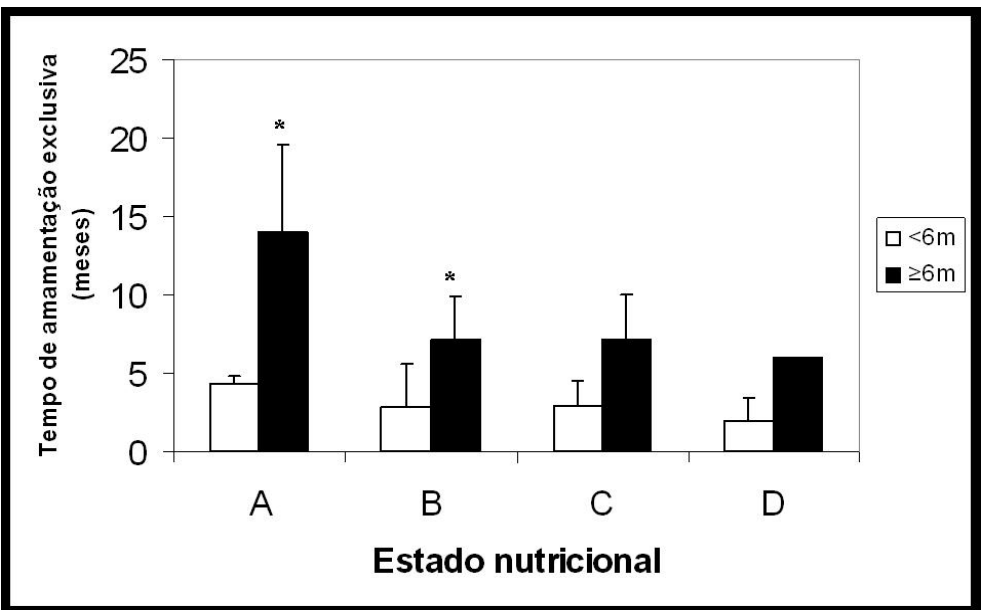

Figura 2.1 - Relação entre estado nutricional e tempo de amamentação exclusiva em crianças na faixa etária de cinco a nove anos residentes nas comunidades rurais de Diamantina/MG, 2008.

Desta forma, nota-se que há uma diferença significativa do estado nutricional 
de crianças que não foram amamentadas exclusivamente com leite materno até os seis meses de idade, predominando nelas o baixo peso ou a desnutrição.

No estudo de Tonete et al. (2006) foi encontrada uma proporção elevada de crianças amamentadas até 30 dias de vida (91,8\%), porém, aos 6 meses de idade, período no qual é recomendado o aleitamento materno exclusivo para prevenção da desnutrição energéticoprotéica, $52,4 \%$ haviam sido desmamadas. Esses resultados demonstram que grande parte do grupo estudado estava exposta ao risco nutricional de baixo peso e desnutrição, o que corrobora os resultados encontrados no presente estudo.

Esse exposto é confirmado por Frota et al. (2007) em seu trabalho realizado com crianças desnutridas residentes na periferia de Fortaleza-CE (evidenciando baixa renda), ressaltando que a desnutrição pode ser decorrente do desmame. A impossibilidade de manter o aleitamento materno efetivo e o conseqüente desmame precoce podem estar relacionados à desnutrição. Esta acontece quando a alimentação é escassa, não contém quantidade suficiente de nutrientes que fornecem proteína e energia ou ainda pode ser acarretada por algumas doenças que modificam as necessidades nutricionais da criança.

Segundo os Indicadores e Dados Básicos (IDB-2009) do Datasus, a taxa de prevalência de aleitamento materno no Brasil, foi de 88\%, no primeiro mês de amamentação e 85,7\%, 83\%, 80,1\%, 76,6\% e $72,9 \%$ do segundo ao sexto mês respectivamente, e no nono e décimo segundo meses, 59,7\% e 44,2\%, mostrando que no Brasil a taxa de prevalência de aleitamento materno reduziu no passar dos meses. Nota-se também que nos primeiros seis meses a da taxa de prevalência foi de $15,1 \%$, portanto, menor que nos últimos seis meses $(28,7 \%)$. Observa-se que do sexto ao nono mês a taxa de prevalência caiu para $59,7 \%$, reduzindo $13,2 \%$ em três meses e do nono ao décimo segundo mês a queda foi de $15,5 \%$. Dessa forma, podemos perceber que do primeiro ao décimo segundo mês a taxa de prevalência de aleitamento materno teve um declínio de 43,8\%. Nota-se também que do primeiro ao segundo mês há uma redução de 2,3\% da taxa de prevalência, enquanto que do nono ao décimo segundo mês essa taxa aumenta para $15,5 \%$, mostrando que 
nos últimos meses as mães começam a desistir da amamentação.

Comparando as regiões brasileiras, os dados do IDB-2009 mostram que a prevalência de aleitamento materno na região Norte foi maior que nas demais (91,6\% no primeiro mês e $54,8 \%$ no décimo primeiro); seguida da região Centro-oeste $(90,9 \%$ no primeiro mês e $47,8 \%$ no décimo segundo), Nordeste $(86,7 \%$ no primeiro mês e $38,8 \%$ no décimo segundo), Sudeste (84,3\% no primeiro mês e 38,6\% no décimo segundo) e por último a região Sul com a menor taxa de prevalência de aleitamento materno $(82,8 \%$ no primeiro mês e $37,2 \%$ no décimo segundo).

Quanto aos dados do IDB-2009 de prevalência de aleitamento materno exclusivo no Brasil em 1999, o primeiro mês a taxa de prevalência foi de 53,1\%, reduzindo para 41,4\% no segundo mês, $30, \%, 21,6 \%, 14,7 \%$ e $9,7 \%$, do terceiro ao sexto mês respectivamente. Dessa forma, podemos perceber que do primeiro ao sexto mês a taxa de aleitamento exclusivo teve um declínio de 43,4\%. Nota-se também que do primeiro ao segundo mês há uma redução de $11,7 \%$ da taxa de prevalência, enquanto que do quinto ao sexto mês essa taxa diminui para 5\%, mostrando que nos primeiros meses há mais desistência por parte das mães em amamentar quando se trata de aleitamento exclusivo.

Comparando as regiões brasileiras, os dados do IDB-2009 mostram que a região Sul possui a maior taxa de prevalência de aleitamento materno exclusivo $(64,3 \%$ no primeiro mês e $12,9 \%$ no sexto); seguida da região Nordeste $(55,4 \%$ no primeiro mês e $10,7 \%$ no sexto), Norte (53\% no primeiro mês e $9 \%$ no sexto), Centro-oeste (50,5\% no primeiro mês e $7,9 \%$ no sexto) e por último a região Sudeste com $42,8 \%$ no primeiro mês e 8,3\% no sexto. Nota-se que a redução da prevalência de aleitamento materno exclusivo é mais significativa nos primeiros meses (primeiro ao terceiro) que nos últimos (terceiro ao sexto)

Os resultados encontrados não apresentaram correlação significativa para eutrofia e sobrepeso em crianças que não foram amamentadas exclusivamente até os seis meses de idade, embora relatos na literatura apontam significância no sobrepeso das crianças. Esse fato pode ter acontecido devido ao pequeno número de 
crianças $(3,4 \%)$ com sobrepeso. No estudo de Balaban et al. (2004), dentre as 409 crianças estudadas, $11,5 \%$ nunca havia recebido leite materno. A duração média do aleitamento materno foi de 3,97 meses. A prevalência de sobrepeso foi de 18,6\%. 0 sobrepeso foi mais prevalente entre as crianças que receberam leite materno exclusivo por menos de quatro meses $(22,5 \%)$ do que entre aquelas que receberam leite materno exclusivo por quatro meses ou mais $(13,5 \%)(p=0,03)$. Concluindo que o aleitamento materno apresentou um efeito protetor contra o sobrepeso em crianças eutróficas.

0 estudo transversal desenvolvido por Siqueira \& Monteiro (2007), apresenta um risco duas vezes superior de sobrepeso em crianças que nunca receberam aleitamento materno em relação às demais crianças, concluindo que estas estão mais sujeitas à ocorrência de sobrepeso e obesidade na idade escolar. Aliado a este relato, os estudos de Liese et al. (2001), Hediger et al. (2001), assim como o de Balaban \& Silva (2004) mostraram resultados semelhantes.
Para dar prosseguimento ao trabalho, fez-se uma análise comparativa das variáveis renda familiar, escolaridade da mãe e número de membros na família em função do estado nutricional das crianças. Pôde-se observar que não houve diferença significativa para a variável renda familiar, já que o p-valor encontrado na análise foi de 0,5123, maior que o nível 0,05 . Como ilustra a figura 3, a maior parte da população avaliada possuía renda familiar menor que dois salários mínimos, evidenciando um predomínio de baixa renda.

Ribas et al. (1999), em seu estudo em relação ao estado nutricional de crianças na região Centro Oeste do Brasil, encontrou associação de desnutrição segundo a renda familiar, mostrando que quanto menor a faixa de renda per capita maior a prevalência de desnutrição, sendo que famílias com menor renda per capita (de 0 a 1 salário mínimo) apresentou prevalência de 9,9\% de déficit de altura/idade (A/I), famílias com renda de 1 a 2 salários mínimos houve prevalência de 2,0\% de déficit de A/I e 1,5\% de déficit para a maior faixa de renda per capita familiar (maior que 2 salários mínimos). No mesmo estudo, segundo o 
índice peso/idade, 90,5\% dos desnutridos pertenciam à faixa de menor renda per capita familiar e 9,5\% à faixa de maior renda. Os estudos de Engstrom \& Anjos (1999) e de Guimarães et al. (1999) corroboram esses dados, encontrando resultados semelhantes.

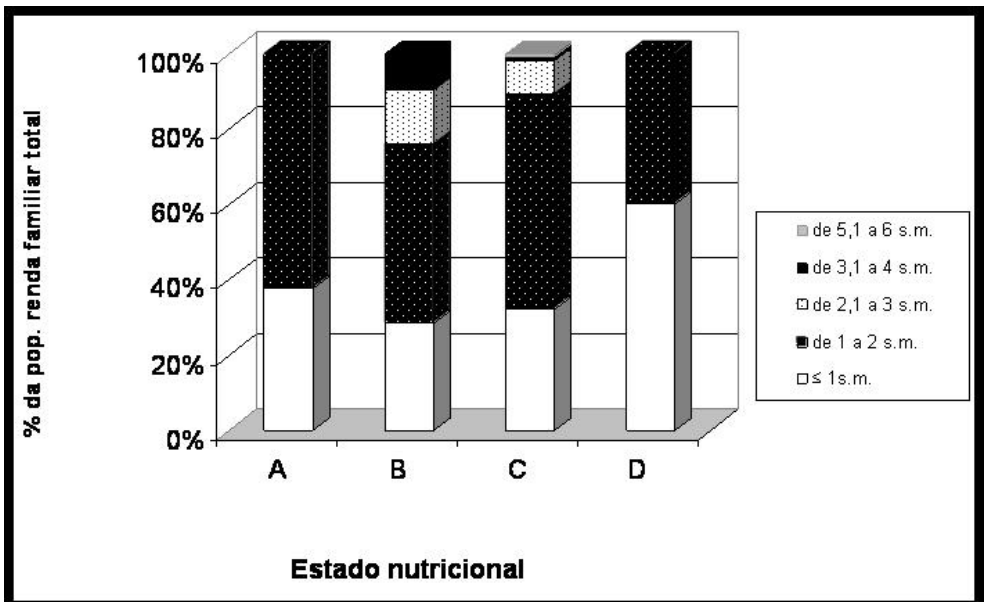

Figura 3- Relação entre o estado nutricional e a renda familiar de crianças na faixa etária de cinco a nove anos residentes nas comunidades rurais de Diamantina, MG, 2008. s.m.=salário mínimo.

Não encontramos nível de significância entre a renda familiar e o estado nutricional das crianças estudadas, como o baixo peso e a eutrofia, embora o estudo de Carvalho, et al. (2009) com 132 crianças, na maioria (90\%) de baixa renda no município do estado de São Paulo, contradiz, mostrando que a renda familiar influencia no estado nutricional de crianças que se encontram, na maioria com risco nutricional (baixo peso ou desnutridas) e que os eutróficos encontrados, na verdade, mascaravam o estado nutricional, sendo na verdade crianças com risco nutricional devido as condições de pais desempregados e famílias numerosas, aumentando a diluição do alimento. Acrescenta ainda que para uma criança atingir os padrões internacionais de crescimento (peso para idade) é necessário que a família receba cerca de 1,5 saláriomínimo per capita, o que não acontece em famílias de baixa renda que não chega à metade do que é considerado satisfatório para assegurar o crescimento adequado das crianças.

Os estudos de Lima et al. (2010) descrevem a freqüência de determinantes do estado nutricional infantil no Nordeste do Brasil em 1986-1996-2006. No que se refere à renda familiar, a melhora do estado nutricional foi apenas modestamente favorável entre 1986 e 1996 e excepcionalmente favorável entre 1996 e 2006. Crianças pertencentes à menor classe de poder aquisitivo somavam mais da metade da população infantil em 1996, assim como nessa pesquisa. 
$\mathrm{Na}$ análise comparativa para a variável escolaridade da mãe, foi encontrado um p-valor de 0,0948, mostrando que não houve relação significativa com o estado nutricional das crianças estudadas. Observase na figura 4 que $82,85 \%$ das mães avaliadas cursaram um tempo menor ou igual à quarta série do primeiro grau.

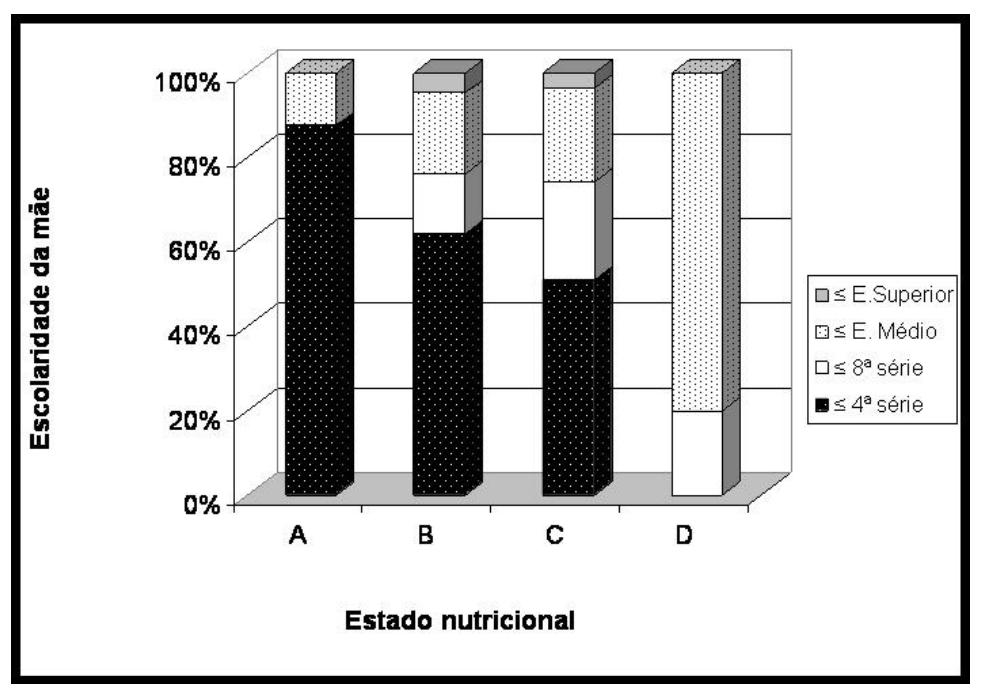

Figura 4- Escolaridade da mãe em função do estado nutricional de crianças das comunidades rurais de Diamantina, 2008. 4a: quarta série do ensino fundamental; 8*a: oitava série do ensino fundamental; E.Médio.: ensino médio; E.Superior.: ensino superior.

Segundo Martins et al. (2007), em seu estudo com interelação de estados nutricionais de crianças pertencentes a famílias de baixa renda em dois municípios de São Paulo, a variável escolaridade da mãe, mostrou-se associada ao déficit estatural em crianças, sendo importante a educação materna na nutrição/crescimento dos filhos, pelo fato da mulher ser a principal provedora de alimentação durante os períodos cruciais do desenvolvimento da criança. Assim, nos primeiros anos de vida, a relação com o meio externo é mediada pela mãe, o que reforça a influência da educação materna no estado nutricional dos filhos. Algo semelhante é encontrado nos estudos de Ribas et al. (1999) em que a prevalência de déficits de altura/idade foi de 7,8\% nas crianças cujos pais possuíam o primeiro grau e nenhum caso nas crianças com pais com segundo grau e 4,3\% ,nas crianças com pais com nível de escolaridade superior. Observou-se que não ocorreram déficits nutricionais de peso/altura em crianças cujo chefe de família possuía curso superior, algo observado também nos estudos de Monteiro (2006) em análise do inquérito "Chamada Nutricional 2005" realizado pelo Ministério do Desenvolvimento Social e Combate à fome e Ministério da Saúde. Os estudos de Engstron \& Anjos (1999) e Guimarães et al.(1999) também encontraram resultados semelhantes. Triches \& Giugliani (2005) 
encontraram resultados significativos para escolaridade da mãe em função do estado nutricional, porém em diferentes resultados desses últimos, observando que a maioria das mães (84\%) que cursaram apenas o ensino fundamental (incompleto e completo) tiveram filhos com sobrepeso de prevalência significativa $(16,9 \%)$.

Já na análise comparativa entre o estado nutricional das crianças estudadas e o número de membros na família, houve diferença significativa. 0 valor encontrado foi de 0,0085 e evidenciou que $83,33 \%$ das famílias era composta por mais de cinco membros e quanto maior o número das pessoas, mais alterado o estado nutricional

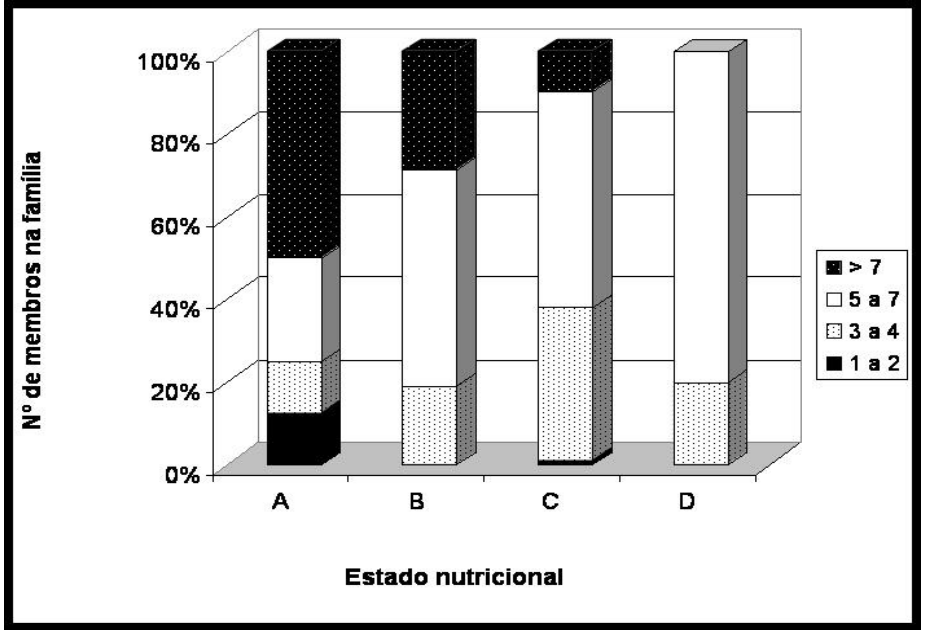

Figura 5- Número de membros na família em função do estado nutricional de crianças das comunidades rurais de Diamantina, 2008.
Na figura 5, pode ser observado que em famílias compostas por 5 a 7 membros, destaca-se o sobrepeso entre as crianças, talvez devido a hábitos alimentares não saudáveis, ricos em carboidratos simples e gorduras. Podemos perceber que $54 \%$ das famílias também possuíam crianças com baixo peso, talvez devido às condições socioeconômicas e higiênico-sanitárias desfavoráveis. Nas famílias que tinham mais de 7 membros houve predomínio da desnutrição e baixo peso, evidenciando que em famílias numerosas, onde o poder aquisitivo é baixo, o risco de desnutrição é maior.

Os estudos realizados por Rissin et al. (2006) no meio rural do estado de Pernambuco, encontraram resultados semelhantes aos aqui apresentados, para a variável número de membros na família em função do estado nutricional. Estes autores observaram que o baixo peso e a desnutrição apresentaram-se significativamente associados às famílias numerosas. Desta maneira, ficou evidente a importância da habitação e de suas características dominantes, como marcadores de risco da ocorrência da desnutrição em escolares. Essa 
relação também foi encontrada nos estudos de Guimarães et al. (1999), realizados com escolares que frequentavam a rede pública de um município de São Paulo.

Como pode ser visto nos relatos acima, tanto a renda familiar quanto a escolaridade da mãe tiveram relação significativa com o estado nutricional de crianças, o que não ocorreu na pesquisa em questão, provavelmente devido ao predomínio de baixa renda na população estudada.

\section{CONCLUSÕES}

Os resultados deste estudo colocam em evidência o perfil do estado nutricional de crianças, na faixa etária entre cinco e nove anos, das comunidades rurais do município de Diamantina/MG. Embora o país esteja vivenciando uma transição nutricional com redução da prevalência de desnutrição e aumento da prevalência de excesso de peso, entre algumas regiões esta não é a norma geral. Neste estudo houve predomínio de crianças em situação nutricional de eutrofia, entretanto, foram encontradas crianças em situação de baixo peso e de desnutrição com valores consideráveis. $\mathrm{Na}$ análise da situação de sobrepeso, a população estudada pode ser considerada como isenta desta situação nutricional.

A desnutrição e o baixo peso remetem as crianças para uma situação de saúde desfavorável, agravada pela situação rural e formação de famílias com baixo poder aquisitivo e baixa escolaridade.

Assim sendo, são necessárias ações para reverter tal quadro, usando medidas preventivas como a promoção do aleitamento materno exclusivo e hábitos alimentares adequados e,ou de intervenções no âmbito social, que devem ser sustentáveis e efetivas para que possam reverter essa situação complexa.

\section{DECLARAÇÃO DE CONFLITO DE INTERESSE}

Os autores declaram não existir quaisquer conflitos de interesse financeiros ou comerciais neste trabalho. 


\section{REFERÊNCIAS}

BALABAN, G.; SILVA, G. A. P.; DIAS, M. L. C. M.; FORTALEZA, G. T. M.; MOROTÓ, F. M. M.;

ROCHA, E. C. V. O aleitamento materno previne o sobrepeso na infância? Revista

Brasileira de Saúde Materno Infantil , v. 4, n. 3, jul/set. 2004.

BISCEGLI, T. S.; POLIS, L. B.; SANTOS, L. M.; VICENTIN, M. Avaliação do estado nutricional e do desenvolvimento neuropsicomotor em crianças freqüentadoras de creche. Revista Paulista de Pediatria, v. 25, n. 4, set. 2007.

BURLAND, Y. L.; ANJOS, L. A. Acesso à alimentação escolar e estado nutricional de escolares no Nordeste e Sudeste do Brasil, 1997. Caderno de Saúde Pública, v. 23, n. 5, mai. 2007

CASTRO, T. G.; NOVAES, J. F.; SILVA, M. R.; COSTA, N. M. B.; FRANCESCHIN, S. C. C.; TINÔCO, A. L. A.; LEAL, P. F. G. Caracterização do consumo alimentar, ambiente socioeconômico e estado nutricional de préescolares de creches municipais. Revista de Nutrição, v. 18, n. 3, mai/jun. 2005.

CARVALHO, L. G.; SALDIVA, S. R. D, M.; Costa ROSA, T. E. C.; LEI, D. L. M. Evolução do estado nutricional de crianças submetidas a um programa de suplementação alimentar em município do Estado de São Paulo.

Revista de Nutrição, Campinas, v. 22, n. 2, p. 207-217, mar./abr. 2009.

CAUÁS, R. C.; FALBO, A. R.; CORREIA, J. B.; OLIVEIRA, K. M. M.; MONTENEGRO, F. M. U.
Diarréia por rotavírus em crianças desnutridas hospitalizadas no Instituto Materno Infantil Prof. Fernando Figueira. Revista Brasileira de Saúde Materno Infantil, v. 6, n. 1, mai. 2006.

DEWEY, K. G. Is breastfeeding protective against child obesity? JHum Lact., v. 19, n. 9, 2003.

FALEIROS, J. J.; KALIL, G.; CASARIN, D. P.; JÚNIOR, P. A. L.; SANTOS, I. S. Avaliação do impacto de um programa de puericultura na promoção da amamentação exclusiva. Caderno de Saúde Pública, v. 21, n. 2, abr. 2005.

FROTA, M. A.; SOUSA, V. R. M.; FILHO, O. A. S.; BARROSO, M. G. T. Diagnósticos das Necessidades Humanas Básicas no contexto sócio-familiar de crianças desnutridas.

Cogitare de Enfermagem, v. 12, n. 2, abr/jun. 2007.

GUIMARÃES, L. V.; LATORRE, M. R. D. O.; BARROS, M. B. A. Fatores de risco para a ocorrência de déficit estatural em préescolares. Caderno de Saúde Pública, v. 15, n. 3, jul/set.1999.

HEDIGER, M. L.; OVERPECK, M. D.; KUCZMARSKI, R. J.; RUAN, J. Association between infant breastfeeding and overweight in young children. JAMA, v. 285, n. 2453, 2001.

KUMMER, S. C.; GIUGLIANIB, E. R. J.; SUSINC, L. O.; FOLLETTOD, J. L.; LERMENE, N. R.; WUF, V. Y. J.; SANTOS, L.; CAETANO, M. B. Evolução do padrão de aleitamento materno. Revista de Saúde Pública, v. 34, n. 2, 2000. 
LAMOUNIER, J. A.; PARIZZI, M. R. Obesidade e Saúde Pública. Caderno de Saúde Pública, v. 23, n. 6, jun. 2007.

LIESE, A. D.; HIRSH, T.; VON, M. E.; KEIL, U.; LEUPOLD, W.; WEILAND, S. K. Inverse association of overweight and breast feeding in 9 to 10 -y-old children in Germany. Int J Obes Relat Metab Disord, v. 252001.

LIMA, A. L. L.; SILVA, A. C. F.; KONNO, S. C.; CONDE, W. L.; BENICIO, M. H. A.; MONTEIRO, C. A. Causas do declínio acelerado da desnutrição infantil no Nordeste do Brasil (1986-1996-2006). Revista de Saúde Pública, São Paulo, v. 44, n. 1, p. 17-27, 2010.

MARQUES, R. F. S. V.; LOPEZ, F. A.; BRAGA, J. A. P. 0 crescimento de crianças alimentadas com leite materno exclusivo nos primeiros 6 meses de vida. Revista chilena de pediatria, v. 77, n. 5 , out. 2006.

MARTINS, I. S.; MARINHO, S. P.; OLIVEIRA, D. C.; ARAÚJO, E. A. C. Pobreza, desnutrição e obesidade: inter-relação de estados nutricionais de indivíduos de uma mesma família. Ciência \& Saúde Coletiva, v. 12, n. 6, 2007.

MONTEIRO, C. A. A dimensão da pobreza, da fome e da desnutrição no Brasil. Estudos Avançados, v. 9, n. 24, 1995.

MONTEIRO, C. A. b. The stage of nutrition in different Brazilian regions.

Archivos Latinoamericano de Nutrición, v. 47, n. 17, 1997.

MONTEIRO, C. A.; CONDE, W. L.; CONNO, S. C. Análise do inquérito "Chamada Nutricional 2005" realizado pelo Ministério do Desenvolvimento Social e Combate à Fome e Ministério da Saúde. Núcleo de Pesquisas Epidemiológicas em Nutrição e Saúde e Departamento de Nutrição da Faculdade de
Saúde Pública da Universidade de São Paulo, 2006.

National Center for Health Statistics - NCHS. Growth curves for children birth-18 years. Vital and Health Statistics series 11, 165 DHEN 781650. Washington D. C. Us: Government Printing Office, 1977.

NHANTUMBO, L.; PRISTA, A.; SARANGA, S.; SEABRA, A.; JÚNIOR, A. L.; MAIA, J. A. R. Avaliação Antropométrica do Estado Nutricional e Aspectos Fragmentados da Realidade Contextual Africana. Revista Brasileira de Cineantropometria e Desempenho Humano, v. 9, n. 3, 2007.

OLIVEIRA, L. P. M.; BARRETO, M. L.; ASSIS, A. M. O.; JUNIOR, A. C. B.; NUNES, M. F. F. P.; OLIVEIRA, N. F.; BENÍCIO, M. H. A.; VENÂNCIO, S. I.; SALDIVA, S. R. M ; ESCUDER, M. M. L. Preditores do retardo de crescimento linear em préescolares: uma abordagem multinível. Caderno de Saúde Pública, v. 23, n. 3, 2007.

POST, C. L.; VICTORA, C. G.; BARROS, F. C.; HORTA, B. L.; GUIMARÃES, P. R. V. Desnutrição e obesidade infantis em duas coortes de base populacional no Sul do Brasil: tendências e diferenciais. Caderno de Saúde Pública, v. 12, n. $1,1998$.

RIBAS, D. B.; PHILIPPI, S. T.; TANAKA, A. C. A.; ZORZATTO, J. R. Saúde e estado nutricional infantile de uma população da região Centrooeste do Brasil. Revista de Saúde Pública, v. 33, n. 4, p. 384-65, Ago. 1999.

RISSIN, A.; FILHO, M. B.; BENÍCIO, M. H. A.; FIGUEIROA, J. N. Condições de moradia como preditores de riscos nutricionais em crianças de Pernambuco, Brasil. Revista Brasileira de Saúde Materno Infantil, v. 6, n. 1, jan/mar. 2006. 
SILVA, R. J. S.; JÚNIOR, A. G. S.; OLIVEIRA, A. C. C. Crescimento em crianças e adolescentes: um estudo comparativo. Revista Brasileira Cineantropometria e Desempenho Humano, v. 7, n. 1, mai. 2005.

SIQUEIRA, R. S.; MONTEIRO, C. A. Amamentação na infância e obesidade na idade escolar em famílias de alto nível socioeconômico. Revista de Saúde Pública, 2007.

SPYRIDES, M. H. C.; STRUCHINER, C. J.; BARBOSA, M. T. S.; K. A. C. G. Amamentação e crescimento infantil: um estudo longitudinal em crianças do Rio de Janeiro, 2001. Caderno de Saúde Pública, v. 21, n. 3, mai. 2005.

TONETE, V. L. P.; FERRIANI, M. G. C.; GUIMARÃES, C. P. Perfil dos beneficiários do Programa de Suplementação Alimentar de uma unidade básica de saúde de Botucatu, São Paulo. Revista de Nutrição, v. 19, n. 4, Jul/Ago. 2006.

TRICHES, R. M.; GIUGLIANI, E. R. J. Obesidade, práticas alimentares e conhecimentos de nutrição em escolares. Revista de Saúde Pública, v. 34, n. 9, 2005.

VASCONCELOS, M. G. L.; LIRA, P. I. C.; LIMA, M. C. Duração e fatores associados ao aleitamento materno em crianças menores de 24 meses de idade no estado de Pernambuco. Revista

Brasileira de Saúde Materno Infantil, v. 6, n. 1, jan/ mar. 2006.
VEIGA, G. V.; BURLANDY, L. Indicadores sócioeconômicos, demográficos e estado nutricional de crianças e adolescentes residentes em um assentamento rural do Rio de Janeiro. Caderno de Saúde Pública, v. 17, n. 6, nov./dez. 2001. 